Pure and Applied Mathematics Quarterly

Volume 6, Number 2

(Special Issue: In honor of

Michael Atiyah and Isadore Singer)

$361-382,2010$

\title{
The Spectral Density Function of a Toric Variety
}

\author{
D. Burns, V. Guillemin and A. Uribe
}

\begin{abstract}
For a Kähler manifold $(X, \omega)$ with a holomorphic line bundle $L$ and metric $h$ such that the Chern form of $L$ is $\omega$, the spectral measures are the measures $\mu_{N}=\sum\left|s_{N, i}\right|^{2} \nu$, where $\left\{s_{N, i}\right\}_{i}$ is an $L^{2}$-orthonormal basis for $H^{0}\left(X, L^{\otimes N}\right)$, and $\nu$ is Liouville measure. We study the asymptotics in $N$ of $\mu_{N}$ for $X, L$ a Hamiltonian toric manifold, and give a very precise expansion in terms of powers $1 / N^{j}$ and data on the moment polytope $\Delta$ of the Hamiltonian torus $K$ acting on $X$. In addition, for a character $k$ of $K$ and the unique unit eigensection $s_{N k}$ for the character $N k$ of the torus action on $H^{0}\left(X, L^{\otimes N}\right)$, we give a similar expansion for the measures $\mu_{N k}=\left|s_{N k}\right|^{2} \nu$. A final remark shows that the eigenbasis $\left\{s_{k}, k \in \Delta \cap \mathbb{Z}^{\operatorname{dim} K}\right\}$ is a BohrSommerfeld basis in the sense of [12], and that the asymptotic results of [1] are exact in this case.

Some of the present results are closely related to earlier results of [10]. The present paper uses no microlocal analysis, but rather an Euler-Maclaurin formula for Delzant polytopes.
\end{abstract}

Keywords: Toric varieties, spectral theory.

\section{Contents}

1. Introduction

Received June 20, 2007.

D.B. supported in part by NSF grant DMS-0514070.

V.G. supported in part by NSF grant DMS-0408993.

A.U. supported in part by NSF grant DMS-0401064. 
2. Toric varieties 366

3. Kähler reduction 368

4. Asymptotics 370

5. Riemann sums 374

6. The Shiffman-Tate-Zelditch results 376

7. Monomials and delta functions 378

$\begin{array}{ll}\text { References } & 381\end{array}$

\section{INTRODUCTION}

The purpose of this note is to explore a fundamental problem in spectral theory in the context of "toric geometry". This problem, formulated in the context of Riemannian geometry, is the following: Let $M$ be a compact Riemannian manifold, and let $\varphi_{i}, i=1,2, \ldots$ be an orthonormal basis of eigenfunctions of the Laplace operator. What can one say about the spectral measures

$$
\mu_{i}=\left|\varphi_{i}\right|^{2} d x
$$

as $i$ tends to infinity? For instance, if the geodesic flow on $T^{*} M$ is ergodic, Schnirelman-Colin de Verdière-Zelditch proved that along "most" subsequences $i_{1}, i_{2}, \cdots, \mu_{i}$ tends weakly to the volume measure, $d x$, [11], [5], [13]. (This phenomenon is known as "quantum ergodicity", and its violation by certain exceptional sequences of $\varphi_{i}$ 's as "quantum scarring".) However, what can one say about the limiting behavior of the $\mu_{i}$ 's if one makes other assumptions about geodesic flow, e. g. that it be periodic or completely integrable? If geodesic flow is periodic then it is known $([7])$ that the eigenvalues of $\sqrt{\Delta}$ clump into clusters,

$$
\lambda_{i, k}, \quad k=1, \ldots, N_{i}
$$

with $\left|\lambda_{i, k}-(a i+b)\right|=O\left(i^{-1}\right)$ (for suitable constants $\left.a, b\right)$, and it is known that a vestige of quantum ergodicity survives: The measures

$$
\nu_{i}=\sum_{k} \mu_{i, k}=\sum_{k}\left|\varphi_{i, k}\right|^{2} d x
$$


tend in limit to the volume measure. (The simplest example is $S^{n-1}$. In this case $\nu_{i}$ is $\mathrm{SO}(n)$ invariant and hence is the volume measure up to a constant factor.)

There is another important instance in which the eigenvalues can be clumped into clusters: If a compact Lie group $K$ acts on $M$ by isometries one can decompose the eigenspaces of $\Delta$ into $K$-invariant subspaces, and consider the spectral measures (1.2), where the $\varphi_{i, k}$ 's are orthonormal bases of these subspaces. If $K$ is an $n$-torus it is also natural to study the asymptotic behavior of the measure (1.1) not for arbitrary sequences of $\varphi_{i}$ 's, but for sequences for which $\varphi_{i}$ lies in a weight space of $K$ of weight $\alpha_{i}$ and the $\alpha_{i}$ tend asymptotically to infinity along a ray in $\mathfrak{k}^{*}$. In both these cases one would like to be able to relate the asymptotics of $\mu_{i}$ and $\nu_{i}$ to properties of the geodesic flow.

These problems have analogues in Kähler geometry: If $X$ is a compact Kähler manifold and $L \rightarrow X$ a Hermitian line bundle whose curvature form is the negative of the Kähler form, then one can consider the asymptotic behavior of the measures

$$
\mu_{N}=\sum\left|\varphi_{N, k}\right|^{2} \nu
$$

where $\left\{\varphi_{N, k} ; k=1, \ldots d_{N}\right\}$ is an orthonormal basis of $\Gamma_{\text {hol }}\left(L^{N}\right)$ (holomorphic sections of $\left(L^{N}\right)$ ) and $\nu$ is Liouville measure. ${ }^{4}$ (Notice that $\mu_{N}$ is now a measure on "phase space". The analogue of $X$ in the case of periodic geodesic flow is the quotient of the unit cotangent bundle of $M$ by the flow.) Using general results about the microlocal structure of Szegö kernels, [2], one can prove that the $\mu_{N}$ have a weak asymptotic expansion as $N \rightarrow \infty$ with leading term Liouville measure.

If there is an action on $X$ of a torus, $K$, preserving the Kähler structure and preserving $L$, one can decompose the spaces $\Gamma_{\text {hol }}\left(L^{N}\right)$ into weight spaces and, as above, study the asymptotics of the measure $\left|\varphi_{N, k}\right|^{2} \nu$ associated with sequences of weights which tend asymptotically to infinity along rays in $\mathfrak{k}^{*}$.

\footnotetext{
${ }^{4}$ This measure can be defined intrinsically as the measure

$$
C^{\infty}(X) \ni f \mapsto \text { Trace } \Pi_{N} M_{f} \Pi_{N}
$$

where $\Pi_{N}$ is the orthogonal projection of $\Gamma\left(L^{N}\right)$ onto $\Gamma_{\text {hol }}\left(L^{N}\right)$, and $M_{f}$ is the operator "multiplication by $f^{\prime \prime}$.
} 
The purpose of this article is to examine both of these problems in the setting of "toric geometry". As a toric variety (together with its canonical Kähler metric) is completely determined by its moment polytope, it is natural to seek results formulated explicitly in terms of the polytope.

In more detail, let $K$ be an $n$-dimensional torus, $X$ a (non-singular) $K$-toric variety, $\phi: X \rightarrow k^{*}$ the moment map associated with the action of $K$ on $X$ and $\Delta=\phi(X)$ the moment polytope. Under the action of $K$ the space $\Gamma_{\text {hol }}\left(L^{N}\right)$ breaks up into an orthogonal direct sum of one-dimensional weight spaces

$$
\Gamma_{\text {hol }}=\bigoplus_{k \in[N \Delta]} \Gamma_{k}
$$

indexed by the set $[N \Delta]$ of integer lattice points in the dilated polytope $N \Delta$, and thus

$$
\mu_{N}=\sum\left\langle s_{k}, s_{k}\right\rangle \nu
$$

where $\left\{s_{k} \in \Gamma_{k} ; k \in[N \Delta]\right\}$ is an orthonormal basis of $\Gamma_{\text {hol }}$ and $\left\langle s_{k}, s_{k}\right\rangle(p)$ is the norm-squared of $s_{k}(p) \in L_{p}^{N}$. Thus to understand the asymptotic behavior of $\mu_{N}$ one has to understand the asymptotic behavior of the functions $\left\langle s_{k}, s_{k}\right\rangle$. Our first step in this direction is the following explicit formula for this function. Let $d$ be the number of facets of the polytope $\Delta$, and $\ell_{i}: \Delta \rightarrow \mathbb{R}, i=1, \ldots, d$, the lattice distance to the $i^{\text {th }}$ facet (see definition 2.1). Then

$$
\left\langle s_{k}, s_{k}\right\rangle=\frac{1}{c_{k}}\left(\phi^{*} \exp \left(N \sum_{i=1}^{d} \ell_{i}\left(\frac{k}{N}\right) \log \ell_{i}-\ell_{i}\right)\right)
$$

where $c_{k}$ is the integral of the expression in the outermost parentheses.

The measure $\mu_{N}$ is $K$-invariant, so it is completely determined by its pushforward to $X / K$. Moreover, $\phi$ is also $K$-invariant, so it defines a map $X / K \rightarrow \Delta$ which for toric varieties is a bijection. Hence to study the asymptotics of $\mu_{N}$ it suffices to study the asymptotics of the measure

$$
\mu_{N}^{\sharp}:=\phi_{*} \mu_{N} .
$$

Moreover, for toric varieties $\phi_{*} \nu$ is just ordinary Lebesgue measure on $\Delta$, hence by (1.4) $\mu_{N}^{\sharp}$ is the measure

$$
\mu_{N}^{\sharp}=\sum_{k \in[N \Delta]} \frac{1}{c_{k}} \exp \left(N \sum_{i=1}^{d} \ell_{i}\left(\frac{k}{N}\right) \log \ell_{i}-\ell_{i}\right) d x .
$$


For $x$ and $y$ in $\Delta$ and $N \in \mathbb{Z}_{+}$let

$$
K_{N}(x, y)=c_{N}(x)^{-1} \exp \left(N \sum_{i=1}^{d} \ell_{i}(x) \log \ell_{i}(y)-\ell_{i}(y)\right)
$$

where

$$
c_{N}(x)=\int_{\Delta} \exp \left(N \sum_{i=1}^{d} \ell_{i}(x) \log \ell_{i}(y)-\ell_{i}(y)\right) d y .
$$

Then, for $f \in \mathcal{C}^{\infty}(\Delta)$,

$$
\int_{\Delta} f d \mu_{N}^{\sharp}=\sum_{k \in[N \Delta]} f_{N}^{\sharp}\left(\frac{k}{N}\right)
$$

where

$$
f_{N}^{\sharp}(x)=\int_{\Delta} K_{N}(x, y) f(y) d y .
$$

One of the main result of this paper is the following.

Theorem 1.1. There exist, for $i=0,1,2, \ldots$, differential operators, $P_{i}(x, D)$ : $\mathcal{C}^{\infty}(\Delta) \rightarrow \mathcal{C}^{\infty}(\Delta)$, of order $2 i$ with the property

$$
f_{N}^{\sharp}(x) \sim \sum_{i=0}^{\infty} P_{i}(x, D)(f)(x) N^{-i} .
$$

Moreover, $P_{0}=I$.

The $P_{i}$ 's are combinatorial invariants of the polytope $\Delta$ (albeit given by rather complicated formulas). By combining this result with an Euler-Maclaurin formula for Riemann sums over polytopes (see [9]), we will be able to write the sum (1.5) as an asymptotic series in inverse powers of $N$ in which the individual terms are integrals over the faces of $\Delta$ of differential expressions in $f$. Moreover, if $k$ is a lattice point of $\Delta$ and $\mu_{N, k}$ is the measure

$$
\mu_{N, k}=\left\langle s_{N k}, s_{N k}\right\rangle \nu
$$

the formula (1.10) yields as a corollary a second main result of this paper:

Theorem 1.2. For $f \in C^{\infty}(\Delta)$ one has an asymptotic expansion

$$
\left.\int_{X} \phi^{*} f d \mu_{N, k} \sim\left(\sum_{i=0}^{\infty} P_{i}(x, D) f N^{-i}\right)\right|_{x=k}
$$


where the $P_{i}$ are the same operators as before.

In case $k$ is in the interior of $\Delta$ this result follows from the results in $\S 7$ and the "matrix coefficients" estimates in [1]. However, we will give below a direct proof that includes the case $k \in \partial \Delta$.

To summarize briefly the contents of this article: In $\S 2$ we will review basic facts about toric varieties, in $\S 3$ derive the formula (1.4), in $\S 4$ prove Theorem 1.1 and in $\S 5$ derive from it the asymptotic expansion mentioned above. The asymptotic properties of $s_{k}$ that we discuss in $\S 4$ are closely related to some results of Shiffman-Tate-Zelditch, and can be viewed as an alternative derivation of these results. (See [10]). We will comment on the relation of our work to theirs in $\S 6$. Also, on the open set where $\left\langle s_{k}, s_{k}\right\rangle$ is non-zero, $-\log \left\langle s_{k}, s_{k}\right\rangle$ is a potential for the Kähler metric on $X$, so inter alia our results give a formula for this Kähler potential in terms of moment polytope data. (For other formulas of this type see [3], [4] and [8].) Finally, in $\S 7$ we show that the basis of sections $\left\{s_{k}\right\}$ is a "Bohr-Sommerfeld basis" in the sense of Tyurin, [12].

It may be worth noting that we make no use of microlocal analysis in this paper.

\section{TORIC VARIETIES}

Let $T$ be the standard $d$-dimensional torus, $T=\left(S^{1}\right)^{d}$, let $\mathfrak{t}=\operatorname{Lie} T=\mathbb{R}^{d}$ and let $e_{1}, \ldots, e_{d}$ be the standard basis vectors of $\mathbb{R}^{d} . T$ acts on $\mathbb{C}^{d}$ by its diagonal action, and if we equip $\mathbb{C}^{d}$ with the Kähler form, $\omega=\sqrt{-1} \sum d z_{i} \wedge d \bar{z}_{i}$ this becomes a Hamiltonian action with moment map

$$
\Psi: \mathbb{C}^{d} \rightarrow \mathfrak{t}^{*}, \quad z \rightarrow \sum\left|z_{i}\right|^{2} e_{i}^{*} .
$$

For $G$ a codimension $n$ subtorus of $T$, let $\mathfrak{g}=\operatorname{Lie} G$, let $\mathbb{Z}_{G}^{*} \subset \mathfrak{g}^{*}$ be the weight lattice of $G$ and let

$$
Q: \mathfrak{t}^{*} \rightarrow \mathfrak{g}^{*}
$$

be the transpose of the inclusion map, $\mathfrak{g} \rightarrow \mathfrak{t}$. Then the action of $T$ on $\mathbb{C}^{d}$ restricts to a Hamiltonian action of $G$ on $\mathbb{C}^{d}$ with moment map

$$
\Phi=Q \circ \Psi=\sum\left|z_{i}\right|^{2} \alpha_{i}
$$


where $\alpha_{i}=Q e_{i}^{*} \in \mathbb{Z}_{G}^{*}$. We will assume that this moment map is proper, or alternatively, that the $\alpha_{i}$ 's are "polarized": for some $\xi \in \mathfrak{g}$, all the numbers, $\alpha_{i}(\xi)$ are positive. The toric varieties we will be considering in this paper are symplectic reduced spaces of the form

$$
X_{\alpha}=Z_{\alpha} / G
$$

where $\alpha$ is in $\mathbb{Z}_{G}^{*}$ and $Z_{\alpha}=\Phi^{-1}(\alpha)$. We recall that the action of $G$ on $\Phi^{-1}(\alpha)$ is locally free iff $\alpha$ is a regular value of $\Phi$, and since we will only be considering non-singular toric varieties in this paper we will assume that $G$ acts freely on $Z_{\alpha}$. Hence $X_{\alpha}$ is a manifold and the projection

$$
\pi: Z_{\alpha} \rightarrow X_{\alpha}
$$

is a principal $G$-fibration. From the action of $T$ on $\mathbb{C}^{d}$ we get a Hamiltonian action of $T$ on $X_{\alpha}$, and if we denote by " $\iota$ " the inclusion of $Z_{\alpha}$ into $\mathbb{C}^{d}$, the moment map for the $T$-action on $\mathbb{C}^{d}$ is related to the moment map $\phi_{\alpha}$ for the $T$ action on $X_{\alpha}$ by the identity:

$$
\phi_{\alpha} \circ \pi=\Psi \circ \iota .
$$

Thus we have a commutative diagram:

$$
\begin{aligned}
& Z_{\alpha} \stackrel{\iota}{\hookrightarrow} \mathbb{C}^{d} \\
& \pi \downarrow \quad \Psi \downarrow \searrow^{\Phi} \\
& X_{\alpha} \stackrel{\phi_{\alpha}}{\longrightarrow} \mathfrak{t}^{*} \stackrel{Q}{\longrightarrow} \mathfrak{g}^{*}
\end{aligned}
$$

The moment polytope for the action of $T$ on $X_{\alpha}$ is

$$
\Delta_{\alpha}=\mathbb{R}^{d} \cap Q^{-1}(\alpha),
$$

by (2.1) and (2.3). (Here we've identified $\mathfrak{t}^{*}$ with $\mathbb{R}^{d}$ via the basis vectors $e_{i}^{*}$.) The facets of this polytope are the intersections of $Q^{-1}(\alpha)$ with the coordinate hyperplanes $x_{i}=0$ in $\mathbb{R}^{d}$.

Definition 2.1. The "lattice distance" to the $i^{\text {th }}$ facet, $\ell_{i}$, is the restriction of the coordinate function $x_{i}$ to $\Delta_{\alpha}$.

Since $G$ acts trivially on $X_{\alpha}$ the action of $T$ on $X_{\alpha}$ is effectively an action of the quotient group, $K=T / G$, and since Lie $K=\mathfrak{k}=\mathfrak{t} / \mathfrak{g}$, the dual $\mathfrak{k}^{*}$ is the annihilator in $\mathfrak{t}^{*}$ of $\mathfrak{g}$, and hence is the kernel of the map $Q$. To make the action of $K$ a Hamiltonian action one has to normalize the moment map, $\phi_{\alpha}: X_{\alpha} \rightarrow Q^{-1}(\alpha)$, so that it maps into $\mathfrak{k}^{*}$, and this one can do by fixing an 
element, $c_{\alpha} \in \mathbb{Z}^{d} \cap \operatorname{Int} \Delta_{\alpha}$ and replacing $\phi_{\alpha}$ by $\phi_{\alpha}-c_{\alpha}$. We won't, however, bother to make this normalization here and will continue to think of $\phi_{\alpha}$ as a map into $Q^{-1}(\alpha)$.

\section{KÄHLER REDUCTION}

In this section we review some general facts about Kähler reduction and use them to derive the formula (1.4). Let $M$ be a complex manifold and $L \rightarrow M$ a holomorphic line bundle. We recall that if $L$ is equipped with a Hamiltonian inner product, there is a unique holomorphic connection on $L$ which is compatible with this inner product. More explicitly, if $\nabla$ is a holomorphic connection and $\langle$,$\rangle an inner product then for every holomorphic trivialization s: U \rightarrow L$

$$
\frac{\nabla s}{s}=\mu \in \Omega^{1,0}(U)
$$

and the compatibility of $\langle$,$\rangle and \nabla$ reduces to

$$
d \log \langle s, s\rangle=\mu+\bar{\mu}
$$

and hence

$$
\partial \log \langle s, s\rangle=\mu
$$

which shows that the inner product determines the connection and vice versa. It also shows that

$$
\operatorname{curv}(\nabla)=\sqrt{-1} \partial \bar{\partial} \log \langle s, s\rangle=:-\omega .
$$

Suppose now that the form, $\omega$, is Kähler. Let $G$ be an $m$-dimensional torus, let $\tau: G \times M \rightarrow M$ be a holomorphic action of $G$ on $M$ and let $\tau^{\sharp}: G \times L \rightarrow L$ be an action of $G$ on $L$ by holomorphic line bundle automorphisms which is compatible with $\tau$. If $\tau^{\sharp}$ preserves $\langle$,$\rangle then by (3.2)-(3.3) it preserves \nabla$ and $\omega$. Moreover, by Kostant's formula there is an intrinsically defined moment map, $\Phi: M \rightarrow \mathfrak{g}^{*}$, such that

$$
L_{v} s=\nabla_{v_{M}} s+i\langle\Phi, v\rangle s
$$

for all $s \in \mathcal{C}^{\infty}(L)$ and $v \in \mathfrak{g}$. In other words the infinitesimal action of $G$ on $\mathcal{C}^{\infty}(L)$ is completely determined by $\Phi$ and $\langle$,$\rangle .$

Let's now describe what symplectic reduction looks like from this Kählerian perspective. Given $\alpha \in \mathbb{Z}_{G}^{*}$, let $Z_{\alpha}=\Phi^{-1}(\alpha)$. Assuming that $G$ acts freely on $Z_{\alpha}$, the reduced space, $X_{\alpha}=Z_{\alpha} / G$ is a $\mathcal{C}^{\infty}$ manifold, and the projection, 
$\pi: Z_{\alpha} \rightarrow X_{\alpha}$ is a principal $G$-fibration. Let $L_{\alpha} \rightarrow X_{\alpha}$ be the line bundle whose fiber at $p$ is the (one-dimensional) space of sections

$$
s: \pi^{-1}(p) \rightarrow L
$$

which transform under $G$ by the recipe

$$
\tau^{\sharp}(\exp v)^{*} s=e^{i \alpha(v)} s,
$$

or alternatively, by (3.5), are auto-parallel along $\pi^{-1}(p)$. For such a section, $\langle s, s\rangle$ is constant along $\pi^{-1}(p)$, so the inner product $\langle$,$\rangle induces an inner product \langle,\rangle_{\alpha}$ on $L_{\alpha}$. In terms of sections, if $\mathcal{C}^{\infty}(L)^{\alpha}$ is the space of global sections of $L$ which transform by (3.6) and $\iota: Z_{\alpha} \rightarrow M$ is the inclusion,

$$
\iota^{*} \mathcal{C}^{\infty}(L)^{\alpha}=\pi^{*} \mathcal{C}^{\infty}\left(L_{\alpha}\right),
$$

and, if $\iota^{*} s=\pi^{*} s_{\alpha}$,

$$
\iota^{*}\langle s, s\rangle=\pi^{*}\left\langle s_{\alpha}, s_{\alpha}\right\rangle_{\alpha}
$$

We now define a complex structure on $X_{\alpha}$ and make $L_{\alpha} \rightarrow X_{\alpha}$ into a holomorphic line bundle by requiring that

$$
\pi^{*} \mathcal{O}_{\alpha} \subseteq \iota^{*} \mathcal{O}
$$

and

$$
\pi^{*} \mathcal{L}_{\alpha} \subseteq \iota^{*} \mathcal{L}
$$

where $\mathcal{O}$ is the sheaf of holomorphic functions on $M, \mathcal{O}_{\alpha}$ the sheaf of holomorphic functions on $X, \mathcal{L}$ the sheaf of holomorphic sections of $L$ and $\mathcal{L}_{\alpha}$ the sheaf of holomorphic sections of $L_{\alpha}$. By (3.3) one gets a holomorphic connection, $\nabla_{\alpha}$, on $L_{\alpha}$ which is compatible with $\langle,\rangle_{\alpha}$ and by (3.4) and (3.8)

$$
\iota^{*} \operatorname{curv}(\nabla)=\pi^{*} \operatorname{curv}\left(\nabla_{\alpha}\right) \text {. }
$$

Thus $-\operatorname{curv}\left(\nabla_{\alpha}\right)$ is the reduced symplectic form on $X_{\alpha}$.

We'll conclude this section by applying these general observations to the set-up in $\S 2$.

Let $L=\mathbb{C} \times \mathbb{C}^{d} \rightarrow \mathbb{C}^{d}$ be the trivial line bundle over $\mathbb{C}^{d}$ and $s: \mathbb{C}^{d} \rightarrow L$ the trivial section, $s(z)=(1, z)$. If we equip $L$ with the Hermitian inner product, $\langle s, s\rangle=e^{-|z|^{2}}$, we get a non-trivial connection on $L$, and by (3.4) the curvature form of this connection is minus the symplectic form, $\sqrt{-1} \sum d z_{i} \wedge d \bar{z}_{i}$. If we let 
$G$ act on $L$ by requiring that $s$ be $G$-invariant then the space $\Gamma_{\text {hol }}(L)^{\alpha}$ is spanned by monomials, $z_{1}^{k_{1}} \ldots z_{d}^{k_{d}}$, for which $\alpha=Q\left(\sum k_{i} e_{i}^{*}\right)=\sum k_{i} \alpha_{i}$, i.e., for which $k \in \mathbb{Z}^{d} \cap \Delta_{\alpha}$. For each of these sections let $s_{k}$ be the corresponding holomorphic section of $L_{\alpha}$. On $Z_{\alpha}$ one has

$$
\left\langle z^{k}, z^{k}\right\rangle=\left|z_{1}\right|^{2 k_{1}} \ldots\left|z_{d}\right|^{2 k_{d}} e^{-|z|^{2}}
$$

and so by (3.8) and (2.1)

$$
\pi^{*}\left\langle s_{k}, s_{k}\right\rangle_{\alpha}=\iota^{*}\left|z_{1}\right|^{2 k_{1}} \ldots\left|z_{d}\right|^{2 k_{d}} e^{-|z|^{2}}=\iota^{*} \Psi^{*}\left(x_{1}^{k_{1}} \ldots x_{d}^{k_{d}} e^{-\sum x_{i}}\right) .
$$

But, by (2.6)

$$
\iota^{*} \Psi^{*}\left(x_{1}^{k_{1}} \ldots x_{d}^{k_{d}} e^{-\sum x_{i}}\right)=\pi^{*} \phi_{\alpha}^{*}\left(\ell_{1}^{k_{1}} \ldots \ell_{d}^{k_{d}} e^{-\sum \ell_{i}}\right) .
$$

Hence we conclude that

$$
\left\langle s_{k}, s_{k}\right\rangle_{\alpha}=\phi_{\alpha}^{*}\left(\ell_{1}^{k_{1}} \ldots \ell_{d}^{k_{d}} e^{-\sum \ell_{i}}\right),
$$

which implies the formula (1.4).

\section{Asymptotics}

In this section we will use stationary phase to analyze the behavior of the integral (1.9) as $N$ tends to infinity. Let

$$
\varphi(x, y)=\sum \ell_{i}(x) \log \ell_{i}(y)-\ell_{i}(y)
$$

be the phase function in this integral. We claim:

Lemma 4.1. For $x$ a fixed point in the interior of $\Delta$, the function $\varphi$ as a function of $y$ has a unique critical point at $x=y$, and this critical point is the unique global maximum of this function on $\Delta$.

Proof. Since

$$
d \varphi=\sum \ell_{i}(x) \frac{d \ell_{i}}{\ell_{i}(y)}-d \ell_{i}=0
$$

at $x=y$, the point $x=y$ is a critical point of $\varphi$ and since

$$
d^{2} \varphi=-\sum \ell_{i}(x) \frac{\left(d \ell_{i}\right)^{2}}{\ell_{i}(y)^{2}}
$$

this critical point is a maximum. Moreover, by (4.2) every critical point of $\varphi$ in the interior of $\Delta$ has to be a maximum and as $y$ tends to the boundary of $\Delta, \varphi$ 
tends to $-\infty$. Hence by the "peaks-passes" lemma $x$ is the only critical point of $\varphi$ and is its unique global maximum.

For $x$ in the interior of a boundary face, $F$, of $\Delta$, one has an analogous result:

Lemma 4.2. The restriction of $\varphi$ to $F$ has a unique critical point at $x=y$, and this critical point is the unique global maximum of $\varphi$ on $\Delta$. In addition, the derivatives of $\varphi$ (as a function of $y$ ) in directions normal to $F$ are not zero at $y=x$.

Proof. Suppose that $F$ is defined by the equations $\ell_{i}=0, i \in I \subset\{1,2, \ldots, d\}$. Then, for $x$ in the interior of $F$,

$$
\varphi(x, y)=\sum_{i \notin I} \ell_{i}(x) \log \ell_{i}(y)-\sum_{i=1}^{d} \ell_{i}(y),
$$

which shows that $\varphi$ is a decreasing function of the $\ell_{i}, i \in I$. The joint minimum of those functions is exactly $F$, and therefore the global maximum of $\Delta \ni y \mapsto$ $\varphi(x, y)$ is attained on $F$. The restriction of this function to $F$ is

$$
\left.\varphi(x, y)\right|_{y \in F}=\sum_{i \notin I} \ell_{i}(x) \log \ell_{i}(y)-\sum_{i \notin I} \ell_{i}
$$

Lemma 4.1 can be applied to this restriction, and therefore $y=x$ is the unique global maximum of $\left.\varphi\right|_{F}$, and so $x$ is the unique global maximum of $\Delta \ni y \mapsto$ $\varphi(x, y)$ on $\Delta$. Moreover, at $y=x$

$$
(d \varphi)_{y}=-\sum_{i \in I}\left(d \ell_{i}\right)_{y}
$$

which shows that the derivatives normal to $F$ are not zero at $x$ (and $\varphi$ decreases to the interior of the polytope).

From these lemmas one obtains the following "localization" theorem for the integral operator defined by (1.10).

Theorem 4.3. Let $f$ and $g$ be in $\mathcal{C}^{\infty}(\Delta)$. Suppose that for $x \in \Delta, f(x) \neq 0$ and $x \notin \operatorname{supp} g$. Then

$$
\left|\int_{\Delta} e^{N \varphi(x, y)} g(y) d y\right| \leq e^{-c N}\left|\int_{\Delta} e^{N \varphi(x, y)} f(y) d y\right|
$$

for some positive constant, $c$. 
We will now examine the local behavior of the transform (1.9) in the neighborhood of a fixed vertex, $p$, of $\Delta$. Let $\Delta_{p}$ be the open subset of $\Delta$ obtained by deleting from $\Delta$ all facets except the facets containing $p$. By repagination we can assume that these are the facets, $\ell_{i}=0, i=1, \ldots, n$.

Lemma 4.4. There exists an affine transform mapping $\Delta_{p}$ onto an open subset of the positive orthant, $\mathbb{R}_{+}^{n}$, mapping $p$ onto the origin and transforming the $\ell_{i}$ 's, $i=1, \ldots, n$, into the coordinate functions, $x_{i}, i=1, \ldots, n$.

(For proof of this "standard fact" about moment polytopes of toric manifolds see [6].)

In these new coordinates the phase function (4.1) takes the form

$$
\varphi(x, y)=\sum x_{i} \log y_{i}-y_{i}+\psi(x, y)
$$

where

$$
\psi(x, y)=\sum_{r>n} \ell_{r}(x) \log \ell_{r}(y)-\ell_{r}(y)
$$

is a $\mathcal{C}^{\infty}$ function on $\Delta_{p}$. Moreover, with $x$ fixed, the derivative of $\psi$ with respect to $y$ :

$$
d \psi=\sum_{r>n} \frac{\ell_{r}(x)}{\ell_{r}(y)} d \ell_{r}-d \ell_{r}
$$

is zero at $x=y$, so

$$
\frac{\partial \psi}{\partial y_{i}}(x, y)=\sum h_{i, j}(x, y)\left(x_{j}-y_{j}\right)
$$

and

$$
\begin{aligned}
\frac{\partial \varphi}{\partial y_{i}} & =\frac{x_{i}-y_{i}}{y_{i}}+\sum_{j} h_{i, j}(x, y)\left(x_{j}-y_{j}\right) \\
& =\frac{1}{y_{i}} \sum_{j}\left(\delta_{i, j}+y_{i} h_{i, j}\right)\left(x_{j}-y_{j}\right) .
\end{aligned}
$$

Hence

$$
x_{j}-y_{j}=\sum g_{i, j}(x, y) y_{i} \frac{\partial \varphi}{\partial y_{i}}
$$

the $g_{i, j}$ 's being $\mathcal{C}^{\infty}$ in a neighborhood of $x=y=0$. 
Consider now an integral of the form

$$
\int_{\mathbb{R}_{+}^{n}} e^{N \varphi(x, y)} f(x, y) d y
$$

where $f$ is $\mathcal{C}^{\infty}$ and supported in a neighborhood of $x=y=0$. Let $\rho(y)$ be a $\mathcal{C}_{0}^{\infty}$ function which is equal to one on a neighborhood of the support of $f$. Then

$$
f(x, y)=\left(f_{0}(x)+\sum\left(y_{j}-x_{j}\right) f_{j}^{\sharp}(x, y)\right) \rho(y),
$$

where

$$
f_{0}(x)=f(x, x)
$$

and

$$
f_{j}^{\sharp}(x, y)=\int_{0}^{1} \frac{\partial}{\partial y_{j}} f(x, x+t(y-x)) d t .
$$

By (4.9) and (4.11) we can write (4.10) as the sum of the two expressions

$$
f_{0}(x) \int_{\mathbb{R}_{+}^{n}} e^{N \varphi(x, y)} \rho(y) d y
$$

and

$$
-\int_{\mathbb{R}_{+}^{n}} e^{N \varphi(x, y)}\left(\sum_{i, j} g_{i, j} f_{j}^{\sharp} y_{i} \frac{\partial \varphi}{\partial y_{i}}\right) \rho(y) d y
$$

and by making the substitution

$$
e^{N \varphi(x, y)} \frac{\partial \varphi}{\partial y_{i}}=\frac{1}{N} \frac{\partial}{\partial y_{i}} e^{N \varphi(x, y)}
$$

and integrating by parts with respect to $y_{i}$ we can rewrite (4.15) in the form

$$
\frac{1}{N} \int_{\mathbb{R}_{+}^{n}} e^{N \varphi(x, y)} f_{1}(x, y) d y
$$

where

$$
f_{1}(x, y)=\sum_{i, j} \frac{\partial}{\partial y_{i}}\left(y_{i} f_{j}^{\sharp} g_{i, j} \rho(y)\right) .
$$

(Notice that in integrating by parts we don't pick up boundary terms because of the presence of the $y_{i}$ 's in the integrand.) 
From (4.14) and (4.16) we get for (4.10) the expansion

$$
f_{0}(x) \int_{\mathbb{R}_{+}^{n}} e^{N \varphi(x, y)} \rho(y) d y+\frac{1}{N} \int_{\mathbb{R}} e^{N \varphi(x, y)} f_{1}(x, y) d y
$$

and by iteration of (4.18), an expansion

$$
\sum_{i=0}^{k-1} f_{i}(x) N^{-i} \int_{\mathbb{R}_{+}^{n}} e^{N \varphi(x, y)} \rho(y) d y+R_{k}(x)
$$

where

$$
R_{k}(x)=N^{-k} \int_{\mathbb{R}_{+}^{n}} e^{N \varphi(x, y)} f_{k}(x, y) d y .
$$

(In more detail: $f_{i}(x)=f_{i}(x, x)$ and $f_{i}(x, y)$ is obtained from $f(x, y)$ by iterating $i$ times the operation (4.17). In particular $f_{i}$ is a sum of derivatives of $f$ of degree less than or equal to $2 i$ with $\mathcal{C}^{\infty}$ functions as coefficients.)

Finally observe that for $x$ near zero the quotient of

$$
\int_{\Delta} e^{N \varphi(x y)}(1-\rho(y)) d y
$$

by

$$
\int_{\Delta} e^{N \varphi(x, y)} d y
$$

is of order $O\left(e^{-c N}\right)$ by Theorem 4.3, hence if we divide the sum (4.19) by (4.21) and let $k$ tend to infinity we get the asymptotic expansion (1.10).

Remark 4.5. In the discussion above we've assumed that $f(x, y)$ is supported on the set $\left\{x, y \in \Delta_{p}\right\}$. However, by the localization Theorem 4.3 one can always reduce to this case by means of a partition of unity.

\section{Riemann SUMS}

One of the many variants of the classical Euler-Maclaurin formula asserts that for $f \in \mathcal{C}_{0}^{\infty}(\mathbb{R})$ the Riemann sum

$$
\frac{1}{N} \sum_{k=0}^{\infty} f\left(-\frac{k}{N}\right)
$$

differs from the Riemann integral

$$
\int_{-\infty}^{0} f(x) d x
$$


by an asymptotic series

$$
\frac{f(0)}{2 N}+\sum_{n=1}^{\infty}(-1)^{n-1} \frac{B_{n}}{(2 n) !} f^{(2 n-1)}(0) N^{-2 n}
$$

where the $B_{n}$ 's are the Bernoulli numbers.

Recalling that

$$
\tau(s):=\frac{s}{1-e^{-s}}=1+\frac{s}{2}+\sum(-1)^{n-1} B_{n} \frac{s^{2 n}}{(2 n) !}
$$

this asymptotic expansion can be written more succinctly in the form:

$$
\frac{1}{N} \sum_{k=0}^{\infty} f\left(-\frac{k}{N}\right) \sim\left(\tau\left(\frac{1}{N} \frac{\partial}{\partial h}\right) \int_{-\infty}^{h} f(x) d x\right)(h=0) .
$$

Guillemin and Sternberg proved in [9] an $n$-dimensional version of this result in which the interval, $(-\infty, 0]$, gets replaced by a convex polytope. In particular for the moment polytopes associated with toric manifolds their formula is basically a "product" version of the formula above and is proved by localization arguments similar to those we used above to prove Theorem 1.1. Let $\Delta \subseteq \mathbb{R}^{n}$ be such a polytope and let $d$ be the number of facets of $\Delta$. Then $\Delta$ can be defined by a set of inequalities

$$
\left\langle u_{i}, x\right\rangle \leq c_{i}
$$

where $c_{i}$ is an integer and $u_{i} \in\left(\mathbb{Z}^{n}\right)^{*}$ is a primitive lattice vector which is perpendicular to the $i^{\text {th }}$ facet and points "outward" from $\Delta$. The Euler-Maclaurin formula in [9] asserts:

Theorem 5.1. Let $\Delta_{h}$ be the polytope

$$
\left\langle u_{i}, x\right\rangle \leq c_{i}+h_{i}, \quad i=1, \ldots, d .
$$

Then for $f \in \mathcal{C}_{0}^{\infty}\left(\mathbb{R}^{n}\right)$

$$
\frac{1}{N^{n}} \sum_{k \in \mathbb{Z}^{n} \cap N \Delta} f\left(\frac{k}{N}\right) \sim\left(\tau\left(\frac{1}{N} \frac{\partial}{\partial h}\right) \int_{\Delta_{h}} f(x) d x\right)(h=0)
$$

where $\tau\left(s_{1}, \ldots, s_{d}\right)=\tau\left(s_{1}\right) \ldots \tau\left(s_{d}\right)$.

Now notice that if we divide (1.8) by $N^{n}$ the right hand side is exactly a Riemann sum of the form above. Hence if we plug in for $f_{N}^{\sharp}$ the asymptotic 
expansion (1.10) and apply to each summand the formula (5.5) we obtain an "Euler-Maclaurin formula" for the asymptotics of the measure $\mu_{N}^{\sharp}$, of the form:

$$
\int_{\Delta} f d \mu_{N}^{\sharp} \sim N^{n} \int_{\Delta} f(x) d x+\sum_{i=1}^{\infty} c_{i}(f) N^{n-i}
$$

where the $c_{i}$ are integrals over the faces of $\Delta$ of differential expressions in $f$.

\section{The Shiffman-Tate-Zelditch Results}

Let $\varphi(x, y)$ be the function (4.1). By (1.4), if $x \in \Delta$ and $k=x N$ is an integral point in $N \Delta$, then

$$
\left\langle s_{k}, s_{k}\right\rangle(p)=\frac{1}{c_{k}(x)} e^{N \varphi(x, y)}
$$

where $p \in \Phi^{-1}(y)$ and

$$
c_{k}(x)=\int_{\Delta} e^{N \varphi(x, y)} d y .
$$

If $x \in \operatorname{Int} \Delta$ then by Lemma 4.1 the function

$$
\Delta \ni y \mapsto \varphi(x, y)
$$

has a unique non-degenerate maximum at $y=x$, and hence by the lemma of steepest descent

$$
c_{k}(x)=\left(\frac{2 \pi}{N}\right)^{n / 2} h(x)^{-\frac{1}{2}} e^{N \varphi(x, x)}\left(1+0\left(N^{-1}\right)\right)
$$

where $h(x)$ is the determinant of the quadratic form

$$
\sum \frac{1}{\ell_{i}(x)}\left(d \ell_{i}\right)^{2}(x) \text {. }
$$

Thus as $k=N x$ tends to infinity along the ray through $x$ one gets the asymptotic identity

$$
\left\langle s_{k}, s_{k}\right\rangle(p) \sim\left(\frac{N}{2 \pi}\right)^{n / 2} h(x)^{1 / 2} e^{N(\varphi(x, y)-\varphi(x, x))}
$$

at $p \in \Phi^{-1}(y)$. In particular, as $N$ tends to infinity $\left\langle s_{k}, s_{k}\right\rangle$ concentrates exponentially on the Bohr-Sommerfeld set $\Phi^{-1}(k / N)$. This result is due to Shiffman, Tate and Zelditch, ${ }^{5}$ who also observe that by applying steepest descent arguments to the function (4.3) one gets an analogue of (6.5) for $x$ lying in the interior of a face, $F$, of $\Delta$. In this case the asymptotic dependence of $\left\langle s_{k}, s_{k}\right\rangle(p)$ on $N$

5 More or less: Their result involves a slightly different choice of inner product on the $s_{k}$ 's and of coordinates on $X$. See [10]. 
is given by an expression similar to (6.5), except that the " $n$ " in (6.5) has to be replaced by the dimension of $F$. Hence the behavior of $\left\langle s_{k}, s_{k}\right\rangle(p)$ for $k=N x$ is very non-uniform in $x$ when $x$ is near the boundary of $\Delta$. We will prove below that by averaging their result over an " $\delta$-pinched" neighborhood

$$
\left|\frac{k}{N}-y\right|<\frac{1}{N^{\delta}}, \quad 0<\delta<\frac{1}{2}
$$

one gets a version of (6.5) which is much more uniform in $k / N$ :

Theorem 6.1. For $x=k / N \in \Delta, \delta \in(0,1 / 2)$, and for every test function $\psi \in C_{0}^{\infty}\left(\mathbb{R}^{n}\right)$,

$$
\int_{\Delta}\left\langle s_{k}, s_{k}\right\rangle \psi\left(N^{\delta}\left(\frac{k}{N}-y\right)\right) d y \sim \sum_{i=0}^{\infty} \sigma_{i}(x) N^{-(1-2 \delta) i},
$$

the $\sigma_{i}(x)$ being $C^{\infty}$ functions on $\Delta$. Thus the averaged estimate, unlike the pointwise estimate (6.5), is "uniform up to the boundary".

Proof. We mimic the integration by parts argument in $\S 4$. Applying this argument to the function

$$
f(x, y)=\psi\left(N^{\delta}(x-y)\right)
$$

and keeping track of powers of $N$ one gets for the integral

$$
\int_{\mathbb{R}_{+}^{n}} e^{N \varphi(x, y)} \psi\left(N^{\delta}(x-y)\right) d y
$$

an expression

$$
\sum_{i=0}^{k-1} \sigma_{i}(x) N^{-i(1-2 \delta)} \int_{\mathbb{R}_{+}^{n}} e^{N \varphi(x, y)} \rho(y) d y+R_{k}(x),
$$

where

$$
R_{k}(x)=N^{-k(1-2 \delta)} \int_{\mathbb{R}_{+}^{n}} e^{N \varphi(x, y)} f_{k}\left(x, y, N^{\delta}(x-y)\right) d y .
$$

Dividing by (4.21) and letting $k$ tend to infinity one gets the estimate (6.6).

Another result of [10] which is closely related to the results of this paper concerns the asymptotic behavior of another interesting measure associated with the norm-squares of the $s_{k}$ 's, namely the measure on the real line

$$
\mathfrak{m}_{N}([t, \infty])=\operatorname{Vol}\left\{\left\langle s_{k}, s_{k}\right\rangle(p) \geq t\right\}
$$


(i. e. the distribution function of the "random variable" $\left\langle s_{k}, s_{k}\right\rangle$ ), where $k=N x$. Assuming that $x$ is a point in the interior of $\Delta$, Shiffman, Tate and Zelditch prove that the moments of this measure have the limiting behavior

$$
\int_{0}^{\infty} t^{m} d \mathfrak{m}_{N} \sim\left(c N^{n / 2}\right)^{m-1} m^{-n / 2}, \quad m=0,1, \ldots
$$

where $c$ is a constant depending on $x$, and from this result deduce that $\mu_{N}$ satisfies "universal rescaling laws" in various regimes (e. g. for $t$ exponentially small with respect to $N$ or for $t$ greater than some positive power of $N$ ). To deduce (6.9) from the results above we note that the integral on the left is just

$$
\int_{X}\left\langle s_{k}, s_{k}\right\rangle^{m} d \nu
$$

where $\nu$ is, as in $\S 1$, Liouville measure. This integral is equal to the integral over $\Delta$ of the right-hand side of $(6.1)$ to the $m^{\text {th }}$ power, with respect to Lebesgue measure. Using (6.2), this gives

$$
\int_{X}\left\langle s_{k}, s_{k}\right\rangle^{m} d \nu=\frac{c_{k m}(x)}{c_{k}(x)^{m}}
$$

But by (6.3)

$$
\frac{c_{k m}(x)}{c_{k}(x)^{m}} \sim\left(\frac{N}{2 \pi}\right)^{\frac{(m-1) n}{2}} m^{-n / 2} h(x)^{\frac{m-1}{2}},
$$

and we recover (6.9). (We are grateful to Zuoqin Wang for pointing out to us this connection between (6.1)-(6.3) and these rescaling laws of [10].)

\section{Monomials and Delta functions}

In this section we present a precise way to relate the sections $s_{k}$ with the BohrSommerfeld fibers of the moment map. This can be seen as a concrete realization of the expected (or hoped-for) equivalence between the complex polarization used in this paper and the singular real polarization defined by such fibers. The result is exact (not asymptotic), so in this section $N=1$.

Let $P \subset L^{*}$ be the unit circle bundle, which is a principal $S^{1}$ bundle with connection. We denote by $\mathcal{H} \subset L^{2}(P)$ the $L^{2}$ closure of the space of smooth functions that extend holomorphically to the unit disk bundle of $L^{*}$, and let $\Pi: L^{2}(P) \rightarrow \mathcal{H}$ be the orthogonal projection. Under the circle action $\mathcal{H}$ splits into isotypical subspaces,

$$
\mathcal{H}=\widehat{\oplus}_{N} \mathcal{H}_{N}
$$


Specifically, $\mathcal{H}_{N}$ consists of eigenspace of the infinitesimal generator of the $S^{1}$ action in $\mathcal{H}$ corresponding to the eigenvalue $\sqrt{-1} N$. $\mathcal{H}_{1}$ is naturally isomorphic with the space of holomorphic sections of $L$. If $s: X \rightarrow L$ is such a section, we will denote by

$$
s^{b} \in \mathcal{H}_{1}
$$

the corresponding function on $P$.

Since the torus $K$ acts on the bundle $L \rightarrow X$ (preserving the hermitian structure) it acts on $P$, preserving the connection. The infinitesimal action of $K$ on $P$ is given by the Kostant formula, (3.5), translated into this setting:

$$
\forall A \in \mathfrak{k} \quad \xi_{A}^{\sharp}=\tilde{\xi}_{A}+H_{A} \partial_{\theta} .
$$

Here:

(1) $\xi_{A}$ is the vector field on $X$ induced by $A$,

(2) $\tilde{\xi}_{A}$ is the horizontal lift of $\xi_{A}$, and

(3) $H_{A}$ is the $A$-component of the moment map $X \rightarrow \mathfrak{k}^{*}$, pulled back to $P$.

Note that, since $H_{A}$ is constant along trajectories of $\tilde{\xi}_{A}$, the two fields on the right-hand side of (7.1) commute. Furthermore, the representation of the torus $K$ on $L^{2}(P)$ by translations commutes with the projection, $\Pi$. Therefore, if $A \in \mathfrak{k}$,

$$
\left[\mathcal{L}_{\xi_{A}^{\sharp}}, \Pi\right]=0,
$$

where $\mathcal{L}$ denotes the Lie derivative.

We begin with:

Lemma 7.1. Let $k \in[\Delta]$ be a lattice point. Then there exists a closed submanifold $Y_{k} \subset P$ such that

(1) $Y_{k}$ is horizontal, and the projection, $P \rightarrow X$, restricted to $Y_{k}$ is a diffeomorphism onto $\phi^{-1}(k)$.

(2) The restriction of $s_{k}^{b}$ to $Y_{k}$ is a non-zero constant function.

Proof. The inverse image $\phi^{-1}(k)$ is an orbit of $K$, and therefore diffeomorphic to a quotient torus, $K / K_{k}$, where $K_{k}$ is the isotropy subgroup of any point in $\phi^{-1}(k)$. The Lie algebra of $K_{k}$ is the conormal space to the face, $F$, of $\Delta$ such that $k \in \operatorname{Int}(F) . \phi^{-1}(k)$ is an isotropic submanifold of $X$, and so a closed horizontal lift, $Y_{k}$, will exist if the holonomies of generators of the fundamental group of 
$\phi^{-1}(k)$ are trivial. By the properties of Delzant polytopes, we can represent generators of $\pi_{1}\left(\phi^{-1}(k)\right)$ by orbits of one-parameter subgroups $\exp (t A)$ of period $T=2 \pi$, and with $A \in \mathfrak{k}$ integral.

Fix $p \in P$ above $\phi^{-1}(k)$, and note that the curve $\exp (t A) \cdot p$ is $2 \pi$ periodic (we are denoting the action of $K$ on $P$ by a dot). Therefore

$$
p=\exp (2 \pi A) \cdot p=\exp \left(2 \pi \tilde{\xi}_{A}\right) \circ \exp \left(2 \pi H_{A}(p) \partial_{\theta}\right)(p) .
$$

Since $A$ and $k$ are integral, $H_{A}(p)=\langle A, k\rangle \in \mathbb{Z}$, and therefore $\exp \left(2 \pi H_{A}(p) \partial_{\theta}\right)(p)=$ $p$. Therefore

$$
p=\exp \left(2 \pi \tilde{\xi}_{A}\right)(p),
$$

that is, the holonomy of an orbit of $\exp (t A)$ in $\phi^{-1}(k)$ is trivial. This proves (1).

To prove (2), note that $\forall A \in \mathfrak{k}$ the section $s_{k}$ satisfies

$$
\mathcal{L}_{\xi_{A}^{\sharp}}\left(s_{k}^{b}\right)=\sqrt{-1}\langle A, k\rangle s_{k}^{b} .
$$

Taking into account that $\mathcal{L}_{\partial_{\theta}} s_{k}^{b}=\sqrt{-1} s_{k}^{b}$, we obtain using (7.1) that $\mathcal{L}_{\tilde{\xi}_{A}}\left(s_{k}^{b}\right)=0$ at points over $\phi^{-1}(k)$. Since this is true $\forall A \in \mathfrak{k}, s_{k}^{b}$ is constant on $Y_{k}$. It is not zero because, as we have seen, $\left\langle s_{k}, s_{k}\right\rangle$ is in fact maximal on $\phi^{-1}(k)$.

The main result of this section is:

Proposition 7.2. Let $k \in[\Delta]$ and $Y_{k} \subset P$ as in the previous proposition. Let $\nu$ be the lift to $Y_{k}$ of a $K$-invariant density on $\phi^{-1}(k)$. Then the projection, $\Pi_{1}\left(\delta_{Y_{k}}\right)$, on $\mathcal{H}_{1}$ of the resulting delta function on $Y_{k}$ is a non-zero constant times $s_{k}$.

Proof. Let $t_{k}=\Pi_{1}\left(\delta_{Y_{k}}\right)$. We begin by clarifying that, as a distribution, $t_{k}$ is defined by the identity

$$
\left(t_{k}, u\right)=\left(\delta_{Y_{k}}, \overline{\Pi_{1}(\bar{u})}\right),
$$

for $u$ a test function on $P$. Therefore, if $u \in \mathcal{H}_{1}$,

$$
\left\langle t_{k}, u\right\rangle_{L^{2}}=\left(t_{k}, \bar{u}\right)=\int_{Y_{k}} \bar{u} \nu
$$

For any $A \in \mathfrak{k}$ let us now compute $\mathcal{L}_{\xi_{A}^{\sharp}}\left(t_{k}\right)$. Using that $\left[\mathcal{L}_{\xi_{A}^{\sharp}}, \Pi_{1}\right]=0$, if $u$ is a test function on $P$

$$
\left(\mathcal{L}_{\xi_{A}^{\sharp}}\left(t_{k}\right), u\right)=-\int_{Y_{k}} \mathcal{L}_{\xi_{A}^{\sharp}}\left(u_{1}\right) \nu
$$


where $u_{1}=\overline{\Pi_{1}(\bar{u})}$. By $(7.1)$ this equals

$$
-\int_{Y_{k}} \mathcal{L}_{\tilde{\xi}_{A}}\left(u_{1}\right) \nu+\sqrt{-1}\langle A, k\rangle \int_{Y_{k}} u_{1} \nu
$$

The first integral is zero, because $Y_{k}$ is horizontal and $\mathcal{L}_{\xi_{A}^{\sharp}} \nu=0$. The second term is

$$
\sqrt{-1}\langle A, k\rangle \int_{Y_{k}} \overline{\Pi_{1}(\bar{u})} \nu=\sqrt{-1}\langle A, k\rangle\left(t_{k}, u\right),
$$

using (7.2). Therefore $\mathcal{L}_{\xi_{A}^{\sharp}}\left(t_{k}\right)=\sqrt{-1}\langle A, k\rangle t_{k}$, that is, $t_{k}$ satisfies the same ODEs as $s_{k}^{b}$, and so necessarily $t_{k}=C_{k} s_{k}^{b}$ for some constant $C_{k}$. To show that this constant is not zero note that

$$
C_{k}=\left\langle t_{k}, s_{k}^{b}\right\rangle_{L^{2}}=\int_{Y_{k}} \overline{\left(s_{k}\right)^{b}} d \nu \neq 0
$$

by (7.3) and part (2) of the previous lemma (in fact $C_{k}$ is equal to the volume of $Y_{k}$ times the conjugate of the constant value of $s_{k}^{b}$ on $Y_{k}$ ).

It is natural to ask if the analogue of the previous proposition holds for other integrable systems on Kähler manifolds, for example the Gelfand-Cetlin system. We hope to return to this problem.

\section{REFERENCES}

[1] D. Borthwick, T. Paul and A. Uribe, "Legendrian distributions with applications to relative Poincaré series", Invent. Math. 122 (1995), 359-402.

[2] L. Boutet de Monvel and V. Guillemin, The spectral theory of Toeplitz operators, Annals of Math. Studies 99, Princeton U. Press, Princeton, NJ, 1981.

[3] D. Burns and V. Guillemin, "Potential functions and actions of tori on Kähler manifolds", Comm. Anal. Geom. 12 (2004) no. 1-2, 281-303.

[4] D. Calderbank, L. David and P. Gauduchon, "About the Guillemin formula for the Kähler potential of a toric manifold", J. Symplectic Geom. 1 (2003), no. 4, 767-784.

[5] Y. Colin de Verdière, "Ergodicité et fonctions propres du Laplacien", Comm. Math. Phys. 102 (1985), no. 3, 497-502.

[6] T. Delzant, "Hamiltoniens periodiques et image convex de l'application moment", Bull. Soc. Math. France, 116 (1988), 315-339. 
[7] J.J. Duistermaat, V.W. Guillemin, "The spectrum of positive elliptic operators and periodic bicharacteristics", Invent. Math. 29 (1975), no. 1, 39-79.

[8] V. Guillemin, "Kähler structures on toric varieties", JDG 40 (1994), 285-309.

[9] V. Guillemin and S. Sternberg, "Riemann sums over polytopes", Ann. Inst. Fourier $\mathbf{5 7}$ (2007), no.7, 2183-2195.

[10] B. Shiffman, T. Tate and S. Zelditch, "Distribution laws for integrable eigenfunctions", Ann. Inst. Fourier (Grenoble) 54 (2004) no. 5, 1497-1546.

[11] A.I. Snirelman, "Ergodic properties of eigenfunctions", Uspehi Mat. Nauk. 29 (1974), no. 6(180), 181-182.

[12] A. N. Tyurin, "On Bohr-Sommerfeld bases" (Russian) Izv. Ross. Akad. Nauk Ser. Mat. 64 (2000), no. 5, 163-196; translation in Izv. Math. 64 (2000), no. 5, 1033-1064.

[13] S. Zelditch, "Uniform distribution of eigenfunctions on compact hyperbolic surfaces", Duke Math. J. 55 (1987), no. 4, 919-941.

\section{Burns}

Department of Mathematics

University of Michigan

Ann Arbor, MI 48109

E-mail: dburns@umich.edu

V. Guillemin

Department of Mathematics

Massachusetts Institute of Technology

Cambridge, MA 02139

E-mail: vwg@mit.edu

A. Uribe

Department of Mathematics

University of Michigan

Ann Arbor, MI 48109

E-mail: uribe@umich.edu 